\title{
DELEUZE: Leibniz... en torno a los pliegues
}

\author{
Deleuze: Leibniz...about the folds
}

\section{Ricardo Espinoza Lolas}

Doctor en Filosofía por la Universidad Autónoma de Madrid y Catedrático de Filosofía Contemporánea de la Pontificia Universidad Católica de Valparaíso. Madrid - Espanha, e-mail: respinoz@ucv.cl

\section{Resumen}

El presente artículo explora la conexión entre dos modos de pensamiento que en apariencia se ven muy distintos, estos son, las filosofías del francés Gilles Deleuze (Paris 1925-Paris 1995) y del alemán Gottfried Leibniz(Leipzig 1646-Hannover 1716). En esta conexión de filosofías se repiensa la posibilidad misma del ejercicio filosófico en la actualidad; es posible que la filosofía se mueva radicalmente en el horizonte de lo barroco y es solamente desde allí donde se da la posibilidad y agenciamiento de la filosofía como disciplina y quehacer humano, en tanto creadora de conceptos, esto es, territorios desde los cuales podemos levantar nuestras formas de vida. Y para poder entender la filosofía como barroca y creadora de conceptos es necesario analizar desde el pensamiento de Deleuze dos categorías fundamentales: pliegue y concepto. Pues desde ellas podemos entender cómo el filósofo francés se siente un fiel heredero de lo más propia de la historia de la filosofía, esto es, la filosofía encarnada por Leibniz. Y desde esta filosofía alemana se dan las herramientas para seguir creando modos nuevos e intensos de estar en la realidad creativamente.

Palabras-clave: Deleuze. Leibniz. Pliegue. Mónada. Concepto. 


\section{Abstract}

The present article explores the connection between(among) two manners of thought that in appearance are seen very different, it these are, the philosophies of the Frenchman Gilles Deleuze (Paris 1925-Paris 1995) and of the German Gottfried Leibniz (Leipzig 1646-Hannover 1716). In this connection of philosophies there is rethought the possibility itself of the philosophical exercise at present; it is possible that the philosophy moves radically in the horizon of the baroque thing and is only from there where it(he,she) gives himself(herself,itself) the possibility and agenciamiento of the philosophy as discipline and human occupation, while creative of concepts, this is, territories from which we prune to raise our forms of life. And to be able to understand the like baroque and creative philosophy of concepts it is necessary to analyze from Deleuze's thought two fundamental categories: plait and concept. Since from them we prune to deal how the French philosopher sits down a faithful inheritor of the most own(proper) of the history of the philosophy, this is, the philosophy personified by Leibniz. And from this German philosophy they give themselves the tools to continue creating new and intense manners of being in the reality creativamente.

Keywords: Deleuze. Leibniz. Fold. Monada. Concept.

\section{INTRODUCCIÓN}

Hay algo espantoso en Leibniz. Es el filósofo del orden. Más aún, del orden y de la policía, en todos los sentidos de la palabra policía. Sobre todo en el primer sentido: la organización ordenada de la ciudad. Sólo piensa en términos de orden. En este sentido es extremadamente reaccionario, es el amigo del orden. Pero muy extrañamente, en este gusto por el orden y para fundar ese orden, se libra a la más demente creación de conceptos más exuberantes, los más desordenados, los más complejos, para justificar lo que es. Es preciso que cada cosa tenga una razón. (DELEUZE, 2006, p. 20). 
Estas palabras son de Deleuze; son de la primera sesión de su curso sobre Leibniz del día 15 de abril de 1980. Del curso del semestre de verano de 1980, y en especial del curso del semestre de invierno de 1986 y 87 que realizó el filósofo en la Universidad de Vincennes (Paris 8), salió posteriormente su notable libro El pliegue. Leibniz y el Barroco (Le Pli Leibniz et le baroque, Les éditions de Minuit (Coll. Critique, Paris, 1988, 191 p.). Leibniz es el "creador de conceptos" por antonomasia para Deleuze, esto es, es el filósofo en todo el sentido profundo del término.

Para este artículo hemos centrado nuestro interés solamente en ciertas obras de Deleuze, pues la impronta de Leibniz en su obra ya de modo explícito como implícito es permanente; estás obras son la ya nombrada El pliegue. Leibniz el Barroco de 1988 y ¿Qué es la filosofía?, de 1991 que escribió junto a su gran amigo Félix Guattari. Pero el texto fundamental para este estudio son las clases que Gilles Deleuze realizó sobre Leibniz en la década de los 80, que fueron sus últimas clases públicas en la universidad, y que han sido transcritas y traducidas directamente del francés de las cintas grabadas que se conservan; y que han sido publicadas en castellano recientemente bajo el nombre: Exasperación de la filosofía, el Leibniz de Deleuze (DELEUZE, 2006, p. 390).

\section{Leibniz, "el” filósofo creador de conceptos}

Leibniz es el filósofo que nos permite entender lo que el pensador francés nos indica con el vocablo "filosofía”. Por este motivo, Deleuze nos indica tajantemente que:

Leibniz es uno de los filósofos que permite comprender de la mejor manera una posible respuesta a esta pregunta: ¿Qué es la filosofía, qué es lo que hace un filósofo, de qué se ocupa? [...] Quisiera decir muy rápidamente cómo reconozco un filósofo en su actividad. No podemos confrontar las actividades más que en función de lo que crean y de su modo de creación. Hay que preguntar qué crea un carpintero, qué crea un músico, qué crea un filósofo. Un filósofo es para mí alguien que crea conceptos. Esto involucra muchas cosas: que el concepto sea algo por crear, que el concepto sea el término de una creación. (DELEUZE, 2006, p. 18).

Vayamos por pasos contados, puesto que entender lo que Deleuze piensa de Leibniz no es algo menor. Interpretar a un filósofo y, en especial a Leibniz, es comprender acabadamente lo que Deleuze entiende por filosofía; lo 
cual no es del todo sencillo. La filosofía no es pensada como "amor a la sabiduría”, esto es, a la verdad; no se trata de buscar las esquirlas más radicales de lo que sean las cosas. ¡No! La filosofía no tiene nada que ver con desentrañar algún rasgo "trascendental” de lo que sean las cosas. La filosofía, por decirlo de alguna manera clásica, no es investigación de lo que sean las cosas en tanto que son; no es metafísica pero tampoco un concordar con un originario (sea como sea como se entienda esto); pues no hay originario alguno al que se pueda por alguna forma llegar; sino mera inmanencia: la vía heideggeriana no es posible (LOLAS; LANDAETA, 2007). Por una parte, la filosofía se entiende desde el filósofo y en esto se le entiende como un creador de conceptos y, por otra, la filosofía se ve a sí misma como una actividad. La filosofía como creadora de conceptos. ¿Qué es lo que crea la filosofía? ¿Conceptos? ¿Qué son los conceptos? ¿Formas de vida? Y, en definitiva, ¿qué se entiende por el papel del filósofo como creador de conceptos en nuestros tiempos?

Deleuze nos dice de la filosofía:

[...] la filosofía es el arte de formar, de inventar, de fabricar conceptos [...] El filósofo es un especialista en conceptos, y, a falta de conceptos, sabe cuáles son inviables, arbitrarios o inconsistentes, cuáles no resisten ni un momento, y cuáles por el contrario están bien concebidos y ponen de manifiesto una creación incluso perturbadora o peligrosa. (DELEUZE; GUATTARI, 2005, p. 8-9).

La filosofía es una actividad como el arte, en tanto creación de conceptos; donde sus obras de arte, son formas de pensamiento, esto es, formas de vida, de vivir con las cosas; los conceptos , como un fabricante de conceptos es lo que propone Deleuze. Entonces ese filósofo, por una parte, es un artista por excelencia; es un formador, inventor, creador de conceptos y, por otra, es crítico y ácido juez de conceptos mal establecidos, rudimentarios, toscos, malhechos; formas en cubierta de negar la vida, de alienarla, de normalizarla, de organizarla, de hacerla descender; ese es el filósofo por excelencia, ese es Leibniz; mejor dicho “el” Leibniz de Deleuze o, si se quiere, es Deleuze el que piensa eso del filósofo y lo ve radicalmente en Leibniz.

De allí que Deleuze no solamente al interpretar diga tal o cual cosa de este filósofo determinado sino que lo dice de sí mismo como filósofo en su actividad propia: la de ser creador de conceptos. Y esto, obviamente, también refluye sobre el autor estudiado, pues en Leibniz se da formalmente la filosofía; se podría decir que "Leibniz" es un modo de filosofía que atraviesa la historia y que está más patente que nunca ahora, por ejemplo, en la propia filosofía de 
Deleuze; es decir, Leibniz es más actual en la época de Deleuze que en la del propio “filósofo" Leibniz. Entonces al estudiar a Leibniz se estudia lo más propio de la filosofía (esto es, los conceptos), la propia filosofía de Deleuze (esto es, los acontecimientos) y también la filosofía del propio Leibniz (esto es, las mónadas), pero que quedará ésta siempre siendo en este bucle de significación; tal bucle va de Deleuze a Leibniz para volver a Deleuze. Esto es, en definitiva, ¡no existe un Leibniz an sich! Pero se entiende ¿qué es un concepto para Deleuze? Y ¿qué significa que la filosofía sea creadora de conceptos? Un concepto no es nada tradicional para Deleuze; no tiene nada que ver con algo abstracto o universal o intencional o dado, sino que nos indica algo singular, un acontecimiento, una textura, algo físico, un territorio por poblar:

Un concepto no es en absoluto algo dado [... Y Yo diría que el concepto es un sistema de singularidades extraídas de un flujo de pensamientos [...] Yo me digo que, de alguna manera, los conceptos son cosas completamente vivientes, son realmente cosos que tienen cuatro patas, que se agitan. Son como un color, como un sonido. Los conceptos son tan completamente vivientes, que no pueden existir sin estar en relación con algo que sin embargo parece lo más lejano del concepto: el grito [...] ¿Qué quiere decir que el concepto sería una especie de grito o una especie de forma de grito? (DELEUZE; GUATTARI, 2005, p.18-20).

Respecto de esto, solamente podemos decir algo puntual, pero volveremos sobre ello al final de este estudio. Los conceptos son acontecimientos (LOLAS, 2007, p. 93-112), territorios, singularidades, mesetas, texturas, tejidos, que surgen por la necesidad del filósofo de forjar algo, un centauro que dome el caos, que dé cuenta de él desde un modo de ver, desde un punto de vista, desde una perspectiva; nacen de la creación del filósofo que se sumerge a gritarnos su modo de dar cuenta del flujo en el que está, del plano de inmanencia en el que se mueve. Y es desde este plano, desde esta materialidad, donde se vuelve en loco creador que nos grita sus conceptos que intentan estabilizar, ordenar, fundar, plegar la totalidad desde cierto acontecimiento. El grito es lo propio de la exasperación del concepto (DELEUZE, 2005, p. 51-61).

No sería exagerado, y menos con Deleuze, decir que "el” filósofo por excelencia, en tanto que se las ha con los conceptos (que se despliegan) es Leibniz. ¿Por qué? Se podría pensar que Spinoza, Kant, Nietzsche, Bergson o Foucault serían "más” plenamente filósofos que Leibniz para Deleuze por ciertos "rasgos" conceptuales que son más atractivos para el filósofo francés o que darían más de sí para ciertos problemas que su filosofía busca resolver, pero no 
es así, es Leibniz el filósofo por excelencia en tanto es el creador desmesurado y enloquecido de conceptos, de singularidades, de acontecimientos, de territorios, de puntos de vista, de mónadas que buscan desplegar la totalidad en la totalidad misma: las multiplicidades en las multiplicidades, las mesetas en las mesetas. Y esto, en sentido estricto, que es lo propio de la filosofía y que acontece en la filosofía de Leibniz, porque en Leibniz se da la "expresión conceptual exasperada" de todo un modo de ser, de un modo de estar en lo incierto mismo, es lo propio del pensamiento de Deleuze; por esto, el modo leibniziano no se agota en un tal momento histórico circunscrito al hombre-Leibniz sino que pervive en otras filosofías y que seguirá siendo un constitutivo fundamental para pensar, para crear conceptos, para dar cuenta de las cosas pero siempre desde un punto de vista, desde una meseta, desde una perspectiva singular que acontece y nos estremece.

Leibniz es un creador de conceptos como todo filósofo, pero lo es de múltiples conceptos, múltiples pliegues conceptuales, múltiples mesetas; es, en definitiva, el filósofo creador de conceptos; de allí que sea el creador en exasperación, fuera de sí, enloquecido, el que grita; y en este modo formal de ser, de sentir la filosofía, su propia filosofía es exasperada. Y esta exasperación filosófica es la que expresa materialmente su filosofía en torno a las mónadas, las series, las curvaturas, etc. Es por esto que su filosofía sea la filosofía del Barroco; su "sistema nuevo", expresa el pliegue al infinito, pero no solamente como algo que fue en tal o cual momento histórico sino la condición misma de toda filosofía y de la realidad, si queremos hablar ontológicamente; la filosofía es en sí misma una empresa barroca, un límite que tiende al infinito. Veámoslo como lo señala Deleuze:

Si el Barroco ha instaurado un arte total o una unidad de las artes, lo ha hecho, en primer lugar, en extensión, al tender cada arte a prologarse e incluso a realizarse en el arte siguiente que lo desborda... Esta unidad extensiva de las artes forma un teatro universal que transporta el aire y la tierra, e incluso el fuego y el agua... El arte, en su totalidad, deviene Socius, espacio social, público, poblado de bailarines barrocos. Plegardesplegar, envolver-desarrollar, son las constantes de esta operación, hoy en día como el Barroco. Ese teatro de las artes es la máquina viviente del 'Sistema nuevo', tal como Leibniz la describe, máquina infinita en la que todas las piezas son máquinas, 'plegadas diferentemente y más o menos desarrolladas’. (DELEUZE, 1989, p. 159).

La filosofía en general y la filosofía de Leibniz en especial, son barrocas, esto es, la filosofía es un pliegue al infinito; no estará de más recordar que para Deleuze la filosofía consiste en ser un empirismo trascendental, como 
la llamaba cuando era joven, o sea, la filosofía es la actividad de crear conceptos en despliegue, por esto esos rasgos de plegar-desplegar, de envolver-desarrollar propios de la filosofía, de los conceptos que se despliegan al infinito, de una imaginación creadora, por esta razón, la filosofía sea esencialmente barroca. El barroco como una máquina viviente de creación, de constante imaginación al infinito, máquina que se desarrolla, se desenvuelve, se despliega; estos son los conceptos, los conceptos filosóficos y esto es en esencia Leibniz.

\section{El pliegue}

[...] el mundo del barroco es el mundo del pliegue que va al infinito y que, desde el principio, se diferencia, se desdobla en dos tipos de pliegues [...]En un piso tenemos los repliegues de la materia y en el otro los pliegues en el alma. El piso de los repliegues de la materia es como el mundo de lo compuesto, de lo compuesto al infinito; la materia no termina de replegarse y de desplegarse. El otro piso es el piso de los simples. Las almas son simples, de ahí la expresión 'los pliegues en el alma', en el alma. (DELEUZE, 2006, p. 140).

Es interesante señalar que la descripción del Barroco como un pliegue que va al infinito es, en cierta forma, la concepción misma del pliegue y de la filosofía de Leibniz en tanto filosofía de mónadas; pero también es el rasgo singular y propio de los conceptos y es lo propio de la filosofía de Deleuze, pero también es lo propio de la "metafísica” de Deleuze, esto es, de la realidad. Deleuze al estudiar a Leibniz nos indica su concepción de las cosas, de ese plano de consistencia o inmanencia, de sensaciones, de materialidad, de cuerpo sin órganos que acontece y se abre creativamente. La materia, las cosas son compuestos siempre en movimiento de repliegue y despliegue; de un constante estar en pliegue, pliegue de pliegue de pliegue; la materia es, de suyo, barroca, se despliega al infinito; y las almas al ser simples se nos muestran como pliegues en ella, singularidades que abran puntos de vistas, perspectivas.

Y la pregunta es ¿cómo se relacionan esos dos pisos, esas dos zonas de la realidad? ¿Cómo se articula la materia con el alma? ¿Cómo se da el agenciamiento entre cuerpo y alma? Tendría que haber "algo" en el primer piso, el piso de los repliegues de la materia que nos permita el "acceso" al piso de los pliegues en el alma. Y a lo mejor nos daremos cuenta de que en el fondo no hay dos pisos si no dos modos de concebir los pliegues que por ser ya pliegues nos permite de inmediato tal articulación, pues el pliegue del segundo 
piso, el alma, sería un momento del pliegue del primer piso, esto es, la materia. ¿Qué hay en la materia que da de sí el alma? En cierta forma en la propia materia tendría que haber algo de tipo cualitativo, esto es, infinito que por ser tal ya por sí mismo es lo que posibilita el otro piso, el de las almas. ¿Qué es esa infinitud de la materia? ¿Qué es lo propiamente cualitativo de ella? La respuesta está ya incoada en lo más propio de la materia:

Puesto que es necesario que los dos pisos comuniquen, para responder qué son esos pliegues en el alma habíamos partido de una búsqueda concerniente a un elemento genético ideal de los repliegues de la materia [...] la materia es una potencia que no deja de replegarse... Si hay un tal elemento genético, sin duda ya forma parte del otro piso...nuestra respuesta había sido que el elemento genético de los repliegues de la materia es la curvatura variable o inflexión. En Leibniz el mundo está fundamentalmente afectado de una curvatura [...] Es que la idealidad matemática es curva, una curvatura del universo. Es un tema leibniziano muy profundo[...] la curvatura variable o inflexión va al infinito. (DELEUZE, 2006, p. 141).

Ya tenemos la respuesta para Leibniz, es la propia curvatura variable de la materia, su inflexión, lo que nos permite el tránsito entre las dos zonas de realidad. El elemento genético propio de la materia, al ser ésta, repliegues infinitos, es ser curvatura; la inflexión, esto es, la flexión interiorizante de la materia, este irse radicalmente para dentro, volverse "concha", ser pliegue es lo que permite todos los repliegues. Esta singularidad de la materia es la que abre la posibles rectas en ella, las series se levantan desde estos nudos singulares; en realidad la materia, entendida como universo, sería un tejido de líneas nodales.

Y ¿cómo se pasa de la curvatura o inflexión al "punto de vista”? La inflexión por ser tal in-flexión, esto es, ser un pliegue que se hunde en sí mismo se vuelve centro, vértice, desde dónde sale la perspectiva, la mirada; luego, el punto se nos ha vuelto en punto de vista, punto de construcción de la totalidad, ésta se dice, se ve desde ese centro vértice. La materia en sus múltiples series que la constituyen está infinitamente tocada por estos centros y en rigor son los centro los que dan dicha series de la materia. Con esto ya tenemos unificada la Casa del Barroco pues sus pisos se tocan en estos puntos, centros o, si se quiere. Son estos puntos los que constituyen la propia casa cuerpo-alma:

¿Por qué de la inflexión al punto de vista? Porque la curvatura variable reenvía, del lado de la concavidad de la curva, a centros de curvatura. Así, pues, la curvatura variable es inseparable de vectores de concavidad. Y el centro, comprendido como centro de curvatura variable, es vértice, 
punto de vista. ¿Qué quiere decir que es vértice? Quiere decir que es el lugar de los puntos donde se encuentran las tangentes a cada punto de la curva variable. (DELEUZE, 2006, p. 141).

Leibniz pasa del universo y su curvatura al punto de vista y con esto ya tiene todo para dar con las mónadas. Pues el vértice de esta curvatura radical al volverse punto de vista se nos vuelve en múltiples visiones constitutiva de las cosas. El vértice punto de vista es lo que nos permite ver, es toda la totalidad vista desde ese vértice punto de vista. Si nos percatamos aquí ya estamos en lo que Deleuze entiende por concepto, a saber, un territorio que acontece vivo y que nos lanza en múltiples caminos posibles de creación. Por esta razón estudiar a Leibniz es estudiar lo más propio de la filosofía en general y de Deleuze en especial. El análisis de los puntos de vista es realizado por Deleuze en cuatro pasos. Por lo importante del análisis, colocaremos el texto casi de modo total y allí veremos no solamente lo que Leibniz piensa del punto de vista sino uno de los pilares de la propia filosofía de Deleuze; lo cual nos evitará de entrada cualquier concepción de manual o provisoria o malintencionada que se ha realizado del filósofo francés, pues no es un filósofo del “caos”, de lo relativo o de lo que "todo vale”:

Era preciso que, desde el principio, el universo estuviera afectado de una curvatura variable... Era preciso que el universo fuera afectado de una curvatura variable para que luego la noción de punto de vista sea verdaderamente, concretamente, fundada [...]. El centro de la curvatura variable ya no es un centro en el sentido de centro de un círculo, es decir de una configuración regular; es un sitio, un vértice. Es un vértice en función del cual veo, es algo que da a ver [...].

Primer carácter: me parece que un punto de vista está siempre en relación con una variación o serie. Más aún, en sí mismo potencia de poner en serie, potencia de ordenar, de ordenar los casos [...].

Segundo carácter: el punto de vista no significa que todo es relativo, no significa que todo es relativo a condición de que lo relativo devenga absoluto. Lo que quiero decir es que el punto de vista no indica una relatividad de lo que es visto. Esto resulta del primer carácter: si verdaderamente es potencia de ordenar los casos, potencia de poner en serie los fenómenos, el punto de vista es condición de surgimiento o de manifestación de una verdad en las cosas. No encontraría ninguna verdad si no tienen un punto de vista determinado [...]. Es la curvatura de las cosas lo que exige un punto de vista [...]. En otros términos, no hay verdad si usted no encuentra un punto de vista bajo el cual tal género de verdad es posible [...].

Tercer carácter: el punto de vista no es una perspectiva frontal que permitiría captar una forma en las mejores condiciones; el punto de vista 
es fundamentalmente perspectiva barroca. ¿Por qué? Porque el punto de vista no es nunca una instancia a partir de la cual se capta una forma, es una instancia a partir de la cual se capta una serie de formas en sus pasajes, sea como metamorfosis -pasaje de una forma a otra-, sea como anamorfosis - pasaje del caos a la forma-[...].

Cuarto y último carácter: el punto de vista está afectado de un pluralismo fundamental. Quien dice punto de vista dice pluralidad de puntos de vista... Que el punto de vista sea esencialmente múltiple, que toda filosofía del punto de vista sea pluralista, no quiere decir [...] 'a cada uno su verdad', esto no funda el pluralismo del punto de vista. Al contrario [... ] aquello que lo funda es la potencia de ordenar y de seriar, de seriar una multitud de formas. El punto de vista se abre sobre una serie infinita [...]. Si todo punto de vista es sobre una serie infinita, sobre el mundo, ¿por qué hay muchos puntos de vista? [...] si el mundo está en inflexión y el punto de vista está definido del lado de la concavidad, hay evidentemente una distribución de los puntos de vista alrededor del punto de inflexión; por tanto, hay necesariamente muchos puntos de vista. (DELEUZE, 2006, p. 142-144).

Digamos algo breve sobre estos cuatro caracteres del punto de vista. En general, obviamente, son aplicables no solamente a Leibniz sino a lo propio de toda filosofía y, en especial, a filósofos como Nietzsche, Foucault y el propio Deleuze. Un centro que se vuelve vértice y por ende modo de visión donde la totalidad cobra asiento desde una perspectiva es lo fundamental del decir deleuziano. El punto de vista es lo que ordena, es lo que establece la serie, es el de dónde nace la serie, los casos se aglutinan en tal o cual serie siempre desde un punto de vista; sea el punto de vista y será cómo se articulan luego las relaciones. Por eso el punto de vista nunca es neutral, no da lo mismo un punto a otro. El punto de vista no tiene nada que ver con lo relativo entendido como lo azaroso o arbitrario. El punto de vista es el único modo como la verdad puede darse, esto es, desde una mirada, una perspectiva, desde un cierto punto singular que acontece y que ahí se deposita la totalidad. En el punto de vista acontece ya el análisis del cambio de formas, metamorfosis, o el advenimiento de la forma desde el caos, anamorfosis, pero nunca descripción de una forma noemática ni menos en sí. Y finalmente en el punto de vista se da la multiplicidad, el punto de vista llama múltiples series, funda la diversidad de las series. Y esto es sencillo de entender pues por cada curvatura plegada, a saber, por cada flexión interna, por cada curvatura puntual hay múltiples maneras de tocarla, son infinitas las tangentes que pasan por la inflexión de la curvatura material, hay entonces infinitos puntos de vistas posibles. 
Si hemos seguido el análisis de Deleuze de Leibniz nos surge la pregunta de todas las preguntas ¿por qué se da el pliegue? ¿Por qué se curva? ¿Por qué la realidad tiende a la envoltura, a enrollarse sobre sí misma? Pues desde los puntos de vistas, que son las series, ya tenemos todos los conceptos para dar con la envoltura por excelencia, esto es, la mónada. La mónada como el concepto filosófico que explica la Casa del Barroco es la idea más tremenda, exasperada y enloquecida de Leibniz. Y es ella la que tenemos que mostrar a continuación, pues debemos responder: ¿Por qué algo está plegado?:

[...] es conocido por todos que Leibniz exige para cada cosa una razón. Se trata de una filosofía que él mismo presenta como filosofía de la razón suficiente, todo tiene una razón [...] Leibniz nos dice sí, el universo está afectado de una curvatura [...] ¿De qué sirve estar plegado?... Si las cosas están plegadas es para ser puestas dentro [...] Las cosas no están plegadas más que para estar envueltas, para estar incluidas, para estar puestas dentro[...] El pliegue remite a la envoltura [...] En otros términos, la envoltura es la razón del pliegue[...] La envoltura es la causa final del pliegue [...]. Había que plegar las cosas para meterlas dentro. (DELEUZE, 2006, p. 145-146).

Todo está plegado para ser envuelto; luego la inclusión es la “razón” de la inflexión y ésta de la curvatura del universo. Las actividades del pliegue en tanto repliegue como despliegue reposan en última instancia en la necesidad más de plegar y esta necesidad de plegamiento requiere envoltura; a saber, un meter dentro radical, así como la concha de un caracol. Todo busca ser envuelto, ser simplemente envuelto, desde una galaxia al cerebro todo tiende a los pliegues que se vuelven sobre sí, todo tiende a la radical singularidad que acontece. Y tal como lo dice Deleuze es interesante por un doble motivo. Por una parte, el célebre tema de la "razón suficiente" de Leibniz queda entendido muy plásticamente desde este esquema del envoltorio y del pliegue, pues todo cosa, todo ente, todo despliegue de la materia es tal despliegue solamente por esa envoltura radical. Y, por otra parte, en la concepción de la envoltura hay múltiples caminos por seguir, incluso, obviamente, más allá de Leibniz. Y por eso Deleuze le dedica un estudio en detalle a Leibniz, puesto que de él el filósofo francés podrá luego dar con múltiples puntos de vista y con ello con las mónadas por él buscada, esto es, los acontecimientos. Para Deleuze el mundo de las envolturas y los pliegues es el mundo de Leibniz: 
Es el mundo de Leibniz: lo que está plegado está necesariamente envuelto en algo, de otro modo no estaría plegado. Lo que es plegado, lo que es curvo, no lo es más que para ser envuelto. Envuelto es en latín involvere o implicare, implicado o envuelto es lo mismo. ¿Qué es implicare? Es el estado de pliegue que está envuelto en algo, implicado en algo [...] Lo que está plegado - plicare- por eso mismo está implicare, está puesto en algo[...] lo que está plegado no existe fuera de aquello que lo incluye, lo implica, lo envuelve[...] Lo que está plegado no existe más que como envuelto en algo[...] En otras palabras, lo plegado sólo existe en su envoltura. (DELEUZE, 2006, p. 146-147).

Finalmente estamos a las puertas de la mónada; Deleuze es tajante en su interpretación de Leibniz: "lo que es plegado, lo que es curvo, no lo es más que para ser envuelto”. Todo lo que está plegado, está implicado en algo, en un dentro; aquí ya estamos en ámbitos del juicio analítico tan caro a Leibniz. Todo, la totalidad está en cierta medida ya contenida en su propia "concha". Y hay múltiples envolturas que llevan dentro de sí sus propias implicaciones, las cuales luego se despliegan en sus múltiples puntos de vistas. Ese mundo plegado en cada acontecimiento, en cada singularidad es el mundo de la Monadología. Como cada sujeto, cada acontecimiento es un único, una concha que envuelve al todo desde múltiples visiones y, si es así, entonces ¿para qué ventanas y puertas paras las mónadas, para estos acontecimientos singulares y envolventes?:

El mundo está envuelto en cada sujeto y no existe más que envuelto en cada sujeto. Es en ese sentido que la Monadología nos dirá que los sujetos, son sin puerta ni ventana. No reciben nada del afuera. Ven ustedes por qué no reciben nada del afuera: porque envuelven, incluyen todo lo que tienen[...] el mundo no existe fuera de los sujetos que lo incluyen, que lo envuelven. (DELEUZE, 2006, p. 152).

¿Qué es el mundo? Los múltiples puntos de vistas, las múltiples series envueltas de modo múltiple en las múltiples mónadas. El mundo es un múltiple de múltiples acontecimientos singulares. Esto es la Monadología de Leibniz y esto es el Acontecimientología de Deleuze. De allí que el mundo no exista fuera de las envolturas singulares, el mundo consiste en ser en esa envolturas singulares, en la múltiples envolturas singulares está plegado múltiplemente el mundo. Y como no existe fuera de esos pliegues envueltos luego no tiene sentido que las singularidades múltiples tengan “orificios”. ¿Para qué un agujero en la mónada? Pues la mónada es en sí mismo un programa que lee desde esas series plegadas que la constituyen. Por tanto, solamente nos basta precisar en qué consiste ser 
sujeto, ser mónada, ser un único que envuelve una cierta totalidad y que como tal ser único se lee en sí misma como ese tal todo.

El sujeto es la noción individual que contiene dentro de sí los estados del mundo, sus series, sus puntos de vistas. El concepto singular encierra dentro de sí todos los predicados posibles. No olvidemos que las definiciones que ha dado Deleuze de la filosofía son: empirismo trascendental y la actividad de crear conceptos. Bueno ambas definiciones, como ya hemos dicho, indican lo mismo. El acto de creación que se despliega, que despliega una totalidad, una totalidad siempre abierta pero que está en cierta medida contenida en dicha singularidad. Por esto que:

[...] el mundo es expresado por cada mónada. Sí, y como el mundo no existe fuera de las mónadas que lo expresan, hay que decir que cada mónada tiene una infinidad de pliegues, el mundo está plegado en cada mónada. (DELEUZE, 2006, p. 369).

El mundo como plegado en cada mónada y plegado de modo múltiple es el "sistema nuevo del mundo" de Leibniz, es la Casa del Barroco. Y por esta razón creemos que para Deleuze dicho mundo está más presente ahora que en los propios tiempos de Leibniz. Hoy vivimos en múltiples hogares barrocos, vivimos envueltos en conchas barrocas, desde donde se dan múltiples posibilidades de desarrollo como de destrucción.

\section{CONCLUSIÓN}

\section{Deleuze, intérprete de Leibniz}

Volvamos al asunto del inicio, en realidad no hemos salido de él, pues no salimos de la mónada-acontecimiento de Leibniz-Deleuze que se nos has desplegado a lo largo de este escrito; sus predicados ya estaban implicados en su singularidad; y precisamente en esto consiste la filosofía como actividad creadora de conceptos, en singularidades que se despliegan como territorios y donde ahí mismo nos quedamos a morar, a poblar y a caminar. Y por eso mismo no hemos salido del concepto exasperado creado por Leibniz (su grito) que resuena en el concepto creado por Deleuze, pues es imposible salir de él, pues no hay un "fuera de él", no tenemos ventanas ni puertas que nos permitan creer 
en un fuera del sistema de la Monadología y del Acontecimientología; lo que hay es una matriz que da de sí todas las posiciones posibles en líneas que se fugan, en intensidades que se salen, en fuerzas que fluyen. No hay un fuera de la Casa del Barroco, pues ¿qué sería un estar fuera del Hogar barroco?

A la filosofía se le da la posibilidad de repensar el presente a la luz de los conceptos que va creando en resonancias y disonancias con los conceptos de otras filosofías pasadas, pero en el caso de Leibniz la cosa es más compleja pues sus conceptos exasperados son el carácter conceptual de los conceptos filosóficos. Por esta razón la filosofía de Deleuze resuena connaturalmente con la de Leibniz, se siente en su casa, no puede salir de ella; el pensar deleuziano habita en la Casa del Barroco de Leibniz como un pez en el agua. Los conceptos de Deleuze nadan en el mar de Leibniz, aquellos conceptos viven en este mar y viven de un modo muy especial: reterritorializándolos.

Como el concepto es un territorio vivo, un acontecimiento singular que expresa de una manera la totalidad, un Centauro creado que se echa a correr y va tomando cuerpo, el territorio va desterritorializando a otros territorios, los vacía de sus contenidos para ser reterritorializados posteriormente como nuevos mundos posibles donde habitar. Si nos damos cuenta, la labor de la filosofía no tiene nada que ver, como hemos indicado al comienzo, con la búsqueda de la verdad, sino con algo mucho más simple pero a la vez más radical. La filosofía reterritorializa los territorios luego de desterritorializarlos. Por esto la filosofía es la creación de las creaciones, crea simplemente conceptos-territorios que des-re-territorializan:

La desterritorialización absoluta no se efectúa sin una reterritorialización. La filosofía se reterritorializa en el concepto. El concepto no es objeto, sino territorio. No tiene un Objeto, sino un territorio. Precisamente, en calidad tal, posee una forma pretérita, presente y tal vez futura. (DELEUZE; GUATTARI, 2005, p. 102).

La filosofía y sus territorios, los cuales están siempre curvados dentro de sí, se agencian como posibilidades de creación, una creación agenciada que se activa, se realiza siempre como series que van dando algún sentido para algunos. Esa activación de la filosofía es al infinito pero hace saltar y hace trizas cualquier organización estabilizada en rígidos conceptos que pretender no ser des-re-territorializados (LOLAS, 2006). Hoy más que nunca vivimos en la Casa del Barroco, puesto que vivimos más que nunca plegados infinitamente en múltiples posibilidades de agenciamientos (es cosa simplemente de pensar en la zona territorial de lo virtual y ya quedamos sobre pasados). Estamos plegados, evidentemente, en un universo leibniziano. Y por esta "razón suficiente": 
Seguimos siendo leibnizianos, aunque ya no sean los acordes los que expresan nuestro mundo o nuestro texto. Descubrimos nuevas maneras de plegar como también nuevas envolturas, pero seguimos siendo leibnizianos porque siempre se trata de plegar, desplegar, replegar. (DELEUZE, 1989, p. 177).

\section{REFERENCIAS}

DELEUZE, G. El pliegue. Leibniz y el Barroco. Barcelona: Paidós, 1989.

Francis Bacon: lógica de la sensación. Madrid: Arena Libros, 2005.

Exasperación a la filosofía. Buenos Aires: Cactus, 2006.

DELEUZE, G.; GUATTARI, F. ¿Qué es la filosofía? Barcelona: Anagrama, 2005.

LOLAS, R. E. Deleuze y Zubiri... en torno a una lógica de la impresión. Contrastes: Revista Interdisciplinar de Filosofía, v. 12, p. 93-112, 2007.

Deleuze... 'contra el imperio del mal’. En torno a nuestra situación actual.

Psikeba: Revista de Psicoanálisis y Estudios Culturales, n. 3, 2006. Disponible en: <www.psikeba.com.ar>. Acceso en: 28 nov. 2008.

LOLAS, R. E.; LANDAETA, P. Deleuze y Heidegger... en torno al signo. Revista Observaciones Filosóficas, n. 4, 2007. Disponível em: <www.observacionesfilosoficas.net>. Acceso en: 01 nov. 2008.

Recebido: 02/01/2009

Received: 01/02/2009

Aprovado: 04/04/2009

Approved: 04/04/2009

Revisado: 05/10/2009

Reviewed: 10/05/2009 